\title{
Effect of aggregation on dielectric property of MWCNT/PDMS nanocomposite
}

\begin{abstract}
Highly graphitized nucleophilic group functionalized Multiwall carbon nanotubes are utilized to fabricate PDMS/FMWCNTs nanocomposites with low weight fraction $(0.1 \mathrm{wt} \%)$. The effect of solvents on dispersion of FMWCNTs is studied. THF shows higher dispersion power in comparison with chloroform. The dielectric constant is measured using impedance analyzer in the range of 106-109 Hz. The result indicate, the dielectric property of FMWCNTs/PDMS nanocomposite could be manipulated with proper mixing techniques and adjustment of curing time.
\end{abstract}

Keyword: Chloroform; Dielectric; FTIR; MWCNT; PDMS; Raman spectroscopy; THF; UVVis 